\title{
Biopsia Contralateral en Teratomas Quísticos Benignos de Ovario
}

\author{
INSTITUTO MATERNO INFANTIL \\ BOGOTA, 1968 - 1988 \\ FACULTAD DE MEDICINA \\ UNIVERSIDAD NACIONAL DE COLOMBIA
}

Rodrigo Díaz Llano MSP*

Una de las conductas tradicionales en ginecología, ha sido la de practicar biopsia en cuña del ovario contralateral en las pacientes en quienes se les extirpa un teratoma quístico benigno (TQB) $(1,3)$, por cuanto se acepta universalmente la bilateralidad de este tumor en proporciones que oscilan entre un 10 y un $20 \%$. En Colombia también se ha recomendado el procedimiento (4).

Debido a esta conducta, en el Instituto Materno Infantil (IMI), se han venido practicando cuñas en ovario contralateral, no solamente en TQB que es lo recomendado, sino también en otras operaciones de tumores benignos del ovario. Sin embargo, esta cuña no es un procedimiento inocuo, porque puede dejar como secuela la infertilidad en algunas mujeres, por adherencias tubo-ováricas. En este sentido, Adashi (5) encontró un 7.8\% de adherencias pélvicas post-cuñas de ovario, en pacientes con síndrome de ovario poliquístico y a quienes se les había practicado resección cuneiforme bilateral, como método terapéutico, aún disponiendo de clomifeno. Este autor piensa que las adherencias son atribuibles a la cuña, por cuanto no estaban presentes en la primera intervención. Más aún, en este mismo trabajo se informa que la probabilidad de embarazos ectópicos en mujeres con antecedentes de cuñas de ovarios, alcanza una proporción 8 veces mayor que en la población general, en la cual es de 0.5 a $1 \%$.

Kistner (6) estudió 16 pacientes con infertilidad post-operatoria a cuñas de ovario, por adherencias pélvicas. Cohen (7) encontró evidencia similar, en estudios endoscópicos. También se observa-

Profesor Asistente, Ginecología y Obstetricia. Facultad de Medicina. Universidad Nacional de Colombia. ron adherencias post-cuñas de ovarios en el 34\% y $14 \%$, de los casos observados por Butram (8) y Weinstein (9) respectivamente. Más recientemente, Toaff y colaboradores, reportaron 7 casos de adherencias post-cuñas en pacientes con síndrome de ovarios poliquísticos (SOPQ) (10). Actualmente, ya no se recomienda la resección en cuña de los ovarios en el SOPQ, como se usó en el pasado, por disponer de tratamientos hormonales adecuados y por el riesgo de adherencias (11). En Colombia también se han reportado buenos resultados en el tratamiento del SOPQ con clomifeno (12).

Teniendo en cuenta los riesgos que ofrece este procedimiento, el presente estudio pretende evaluar la utilidad diagnóstica que ofrece la cuña de ovario contralateral en los tumores benignos del ovario, y en especial, en los teratomas quísticos benignos. Además, se desea establecer la proporción de cuñas en las cuales aparece el tumor, para juzgar la validez de una conducta que se está practicando en ovarios de apariencia normal.

\section{MATERIALES Y METODOS}

Para obtener la información necesaria de este estudio, se revisaron todos los protocolos de piezas quirúrgicas del Departamento de Anatomía Patológica del IMI, durante el período comprendido entre el 1o. de enero de 1968, hasta el 31 de diciembre de 1988 (21 años). Se analizaron solamente los quirúrgicos internos, o sea los producidos en el IMI, y no se tuvieron en cuenta los procedentes de otras instituciones. En el lapso descrito se realizaron 57.053 estudios patológicos de especímenes quirúrgicos internos, los cuales fueron revisados. De este conjunto, fueron seleccionados únicamente los ca- 
sos correspondientes a tumores benignos de ovario. Se excluyeron los carcinomas, las ooforectomías por cáncer mamario, los hallazgos casuales de quistes benignos de ovario en histerectomías de causa uterina, los casos de síndrome de Turner, feminización testicular o disgenesias gonadales; tampoco se tuvieron en cuenta las biopsias de ovario en los estudios endocrinológicos, ni las ooforectomías por abscesos tubo ováricos.

De los 57.053 casos, y una vez realizada la exclusión descrita, quedaron 1.162 casos para considerar en el estudio. Bajo el epígrafe de "tumores no neoplásicos" quedaron registrados los quistes foliculares, quistes lúteos, cuerpos amarillos quísticos, y los teca-luteínicos. En "otros", se incluyeron 2 tecomas -19 y 40 años-; un ovario normal -24 años-con su cuña sana; un tumor fibromucinoso -69 años-; y un sero-mucinoso -70 años-. (Para facilitar la presentación de los resultados, se decidió clasificar a los otros tumores mixtos, según el predominio del material contenido, en serosos, mucinosos, o fibromas. Los 2 que se describen aquí no tenían predominio de uno u otro).

\section{RESULTADOS}

Se obtuvieron 1.162 protocolos de patología, de cirugías de ovarios, (ooforectomías o tumorectomías). El Cuadro No. 1 muestra la distribución en el período de estudio, por trienios, de los casos a los cuales se les practicó resección en cuña al ovario contralateral; aunque se aprecia una reducción en la pŕactica de esta conducta en el tiempo, de todas maneras se continúa efectuando en más de la tercera parte de los casos.

\section{CUADRO 1}

DISTRIBUCION DE FRECUENCIAS EN CASOS CON CUNA CONTRALATERAL, EN EL PERIODO 1968-1988

\begin{tabular}{|c||c||c||c|}
\hline ANOS & $\begin{array}{c}\text { TOTAL } \\
\text { DE } \\
\text { TUMORES BENIONOS }\end{array}$ & CURAS PRACTICADAS & $\%$ \\
\hline $1968-1970$ & 157 & 112 & 71 \\
\hline $1971-1973$ & 131 & 72 & 56 \\
\hline $1974-1976$ & 190 & 77 & 40 \\
\hline $1977-1979$ & 143 & 57 & 40 \\
\hline $1980-1982$ & 150 & 54 & 36 \\
\hline $1983-1986$ & 198 & 63 & 32 \\
\hline $1986-1988$ & 196 & 70 & 36 \\
\hline \hline TOTAL & 1.162 & 808 \\
\hline
\end{tabular}

R.DAA OE OVARIO GONTMALATERAL 1888-1.980.
Como puede observarse en el Cuadro No. 2, 313 casos correspondieron a TQB, aun cuando en solo 204, se practicó cirugía contralateral. Además, se observó que del total de casos, se practicó algún tipo de cirugía en ovario contralateral, en general cuñas, a 505 casos $(43,5 \%)$, los cuales se describen en el cuadro No. 4. De los 505 casos con cirugías contralaterales, se encontraron 31 casos de TQB bilaterales; en todos los casos el tumor contralateral era evidente por su tamaño, como se describe en el Cuadro No. 5. Los 3 TQB más pequeños encontrados en el ovario contralateral, tenían en promedio $2 \mathrm{~cm}$. de diámetro.

\section{CUADRO 2}

TIPO DE CIRUGIA PRACTICADA EN TUMORES BENIGNOS DE OVARIO: TUMORECTOMIA SIMPLE VS. TUMORECTOMIA MAS CUÑA CONTRALATERAL

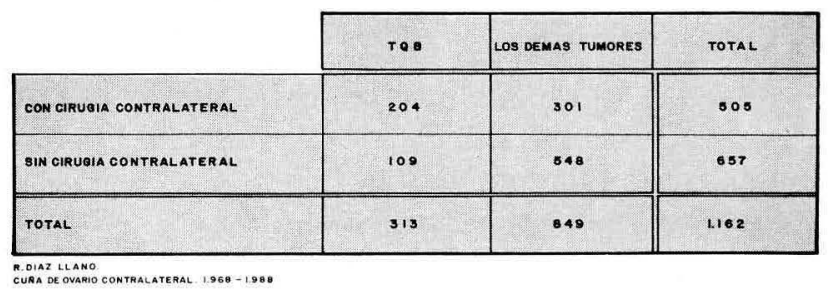

CUADRO 3

CIRUGIA CONTRALATERAL EN TUMORES BENIGNOS DE OVARIO.COMPARACION DE PRESENCIA VS. AUSENCIA DE BILATERALIDAD

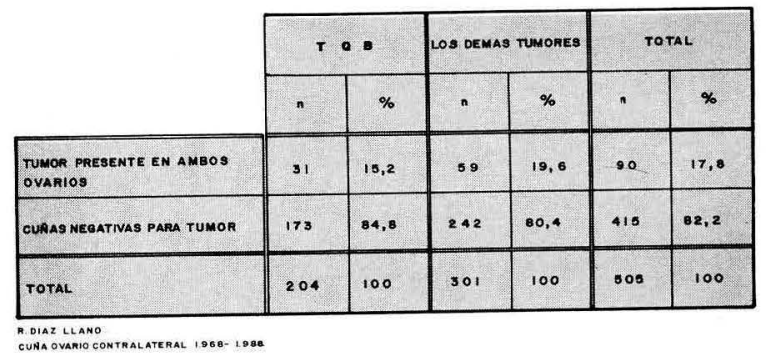

En el Cuadro No. 3 se aprecia que en los 240 casos de TQB a los cuales se les practicó cirugía contralateral, el tumor también estuvo presente en el otro ovario en 31 casos (15,2\% de bilateralidad) y 173 cuñas fueron "negativas para tumor". También se aprecia algo similar en los demás tumores. En general, se puede observar que el $82 \%$ de las cuñas practicadas en ovarios contralaterales resultaron negativas. 
CUADRO 4

DISTRIBUCION DE TUMORES BENIGNOS CON GUNAS GOTRALATERALES, POR GRUPOS DE EDAD 1968-1988

\begin{tabular}{|c|c|c|c|c|c|c|c|c|c|c|c|}
\hline $\begin{array}{c}\text { EDAD } \\
\text { (on afos) } \\
\end{array}$ & TQB & $\begin{array}{l}\text { Cistoden- } \\
\text { serosso }\end{array}$ & $\begin{array}{l}\text { Cistaden - } \\
\text { mucinoso }\end{array}$ & Fibroma & \begin{tabular}{|l|} 
Tr. no \\
Neoplásicos
\end{tabular} & \begin{tabular}{|c|} 
Quistes \\
para \\
Ovarios
\end{tabular} & $\begin{array}{l}\text { Endomor rio- } \\
\text { silo }\end{array}$ & $\begin{array}{l}\text { Clistadeno- } \\
\text { Fibromos }\end{array}$ & $\begin{array}{l}\text { Sindrome } \\
\text { Ovaripas } \\
\text { Poliquiticos }\end{array}$ & OTROS & TOTAL \\
\hline $10-17$ & 8 & 6 & 1 & 4 & 6 & 1 & 1 & 1 & 2 & - & 30 \\
\hline $18-24$ & 55 & 25 & 7 & 4 & 10 & 9 & 3 & 2 & 14 & 2 & 131 \\
\hline $25-29$ & 38 & 19 & 4 & 7 & 9 & 6 & 6 & - & 13 & - & 102 \\
\hline $30-34$ & 34 & 24 & 10 & 6 & 7 & 5 & 2 & - & 3 & - & 91 \\
\hline $35-39$ & 26 & 15 & 3 & 4 & 3 & 4 & 4 & - & - & - & 59 \\
\hline $40-49$ & 25 & 16 & 6 & 1 & 3 & 2 & 2 & - & - & 1 & 56 \\
\hline $50-59$ & 4 & 2 & 2 & - & - & - & - & - & - & - & $\theta$ \\
\hline 60 Y MAS & - & - & - & - & - & 1 & - & - & - & 2 & 3 \\
\hline SIN DATO & 14 & 1 & 2 & 2 & - & 2 & 2 & 2 & - & - & 25 \\
\hline TOTAL & 204 & 108 & 36 & 28 & 38 & 30 & 20 & 5 & 32 & 5 & 508 \\
\hline
\end{tabular}

El Cuadro No. 4 muestra la distribución de los tumores benignos de ovario, y con cuñas sobre el otro ovario, por grupos de edad. Puede apreciarse que hay 30 casos de niñas con edades entre 10 y 17 años, en quienes se practicó una cuña, como procedimiento adicional a la ooforectomía. La distribución de frecuencias de las clases tumorales, corresponde a lo encontrado en otros trabajos $(13,14)$.

En todos los casos de TQB bilateral, el tumor principal (el más grande), tenía en promedio $10 \mathrm{~cm}$ de díametro, siendo los más voluminosos de $29 \mathrm{x}$ $25 \mathrm{~cm}$ y los menores de $5 \times 4 \mathrm{~cm}$. De otra parte, el tumor contralateral tenía suficiente tamaño como para ser detectado con la inspección; los diámetros de estos tumores contralterales aparecen en el Cuadro No. 5 y van desde $10 \mathrm{~cm}$ hasta $2 \mathrm{~cm}$ de diámetro promedio.

Además de los TQB, se encontraron los siguientes casos de tumor en ovario contralateral:

Endometrosis ..............

5 casos

Cistadenoma mucinoso . . . . . . . . 6 casos

Cistadenoma seroso .......... 8 casos

Tumores no neoplásicos ........ 11 casos

Síndrome de ovario poliquístico .... 29 casos

Total

59 casos.

Por otra parte, se encontraron 657 casos de tumores benignos de ovario a los cuales no se les practicó resección contralateral; (éstos, no se incluyen en el Cuadro No. 4). De este grupo, 109 TQB a los cuales no se les practicó cuña contralateral, posiblemente porque el cirujano no lo consideró necesario.

Sin considerar estos últimos casos de TQB (que presumiblemente no tenían bilateralidad), se puede ver que de los 204 casos de teratomas benignos, con cirugía sobre el otro ovario, sólo apareció bilateralidad en 31 casos (15\%), 28 de los cuales tenían más de $4 \mathrm{~cm}$ en su diámetro mayor. Unicamente en 3 casos ( $1.5 \%)$ el tumor contralateral era pequeño, de $2 \mathrm{~cm}$ de diámetro promedio.

\section{DISCUSION}

Actualmente existe evidencia de que las resecciones en cuña del ovario, pueden comprometer la fertilidad de estas pacientes, por la formación de adherencias tubo-ováricas post-operatorias (5 - 10). Esta es una de las razones por la que se ha abandonado el procedimiento en los síndromes de ovarios poliquísticos, además de que se dispone de tratamientos hormonales adecuados $(11,12)$.

CUADRO 5

TERATOMA QUISTICO BENIGNO BILATERAL

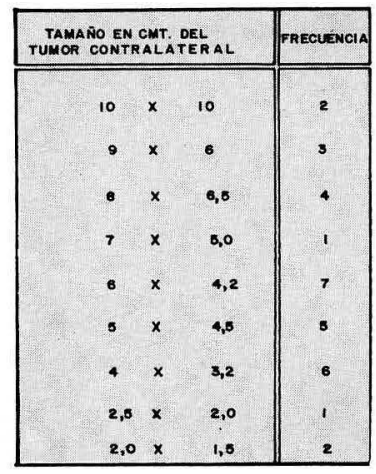


De acuerdo con los hallazgos de este estudio, en nuestro medio se están practicando cuñas en ovario contralateral, en una gran cantidad de tumores benignos de ovario (301 casos), en los cuales no está indicado el procedimiento. La indicación clásica de la cuña ha sido en el TQB $(1,3)$; sin embargo, los resultados muestran que la bilateralidad del tumor $(15 \%)$ ocurre con masas que pueden ser reconocidas con una inspección cuidadosa del ovario, durante la exploración quirúrgica. Aparentemente la cuña no sirve para reconocer tumores microscópicos, y en efecto, los 173 casos de cunas "negativas para tumor" parecen confirmarlo. En los $31 \mathrm{ca}-$ sos restantes en los cuales hubo bilateralidad, el tumor fue macroscópico en todos los casos.

Practicar cuñas de rutina en ovarios aparentemente sanos, puede ser más perjudicial que beneficioso, por cuanto la probabilidad de adherencias pélvicas es mayor ( $8 \%$ ), que la de encontrar tumores imperceptibles a la inspección externa del ovario.

\section{CONCLUSIONES}

En este trabajo, se encontraron 313 casos de TQB, de los cuales 109 fueron resecciones unilaterales, y 204 tuvieron cirugía sobre el ovario contralateral. Se encontró tumor bilateral en 31 casos (15\%), 28 de los cuales tenían un diámetro mayor superior a $4 \mathrm{~cm}$. Además, se practicaron cuñas contralaterales (que resultaron negativas) en 301 casos de otros tumores de ovario.

Con estos resultados, se piensa que se están practicando una cantidad innecesariamente alta de cuñas de ovario, y en los casos de TQB, parece que lo adecuado es la inspección cuidadosa del ovario contralateral, más aún en mujeres jóvenes y con expectativas reproductivas.
Se debería aceptar la recomendación de que en los casos de TQB se observara cuidadosamente el otro ovario por inspección y palpación, y si tiene aspecto normal, se deja intacto, sin cuña, por el riesgo de adherencias e infertilidad posterior (15). En mujeres jóvenes, debería practicarse tumorectomía simple, preservando tejido ovárico, siempre que sea posible.

\section{RESUMEN}

Este trabajo pretende evaluar la utilidad de la biopsia en cuña de ovario contralateral, como procedimiento de rutina en los casos de teratomas quísticos benignos (TQB). Se analizan 21 años de lo ocurrido en el Instituto Materno Infantil de Bogotá, y se encontró que en ese período se operaron 313 casos de TQB. En 109 no practicaron cuña contralateral; pero en los 204 casos restantes si se practicó este procedimiento, encontrándose solo 31 casos de bilateralidad, en los cuales el tumor contralateral fue evidenciable a simple inspección visual, debido a su tamaño. Los 173 casos restantes fueron cuñas "negativas para tumor".

\section{AGR ADECIMIENTOS}

Debo agradecer a la Dra. Joanne Klevens, MSP, por su ayuda en la tabulación de los datos; al Dr. Rafael Gómez R., quien colaboró en la búsqueda de bibliografía no reciente; a los profesores del Departamento de Patología del IMI, ẹ especial a los Doctores Ricardo Alvarado P., Manuel Bustamante, y Odilio Méndez por sus oportunos conceptos, y porque ellos leyeron la inmensa mayoría de las biopsias de ovario, como parte de su trabajo cotidiano.

\section{BIBLIOGRAFIA}

1. CURTIS A.H.; HUFFMAN J.W. A textbook of Gynecology. Philadelphia: Saunders Company, 1950, chapt 23: 415 - 417.

2. KISTNER R.W. Tratado de Ginecología. Barcelona: Ediciones Toray, 1974, pag. 423.

3. MONTOBBIO V.C. Tratado de ginecología y de técnica terapéutica ginecológica. Barcelona: Editorial Labor, 1946. pag. $713-740$.

4. CACERES E, BARBOSA A. Patología ovárica en pacientes mayores de 40 años. Rev Col Obs Ginec. 1968, 3: 209 - 214.

5. ADASHI, E.Y.; ROCK, J.A.; GUZICK, D.; WENTZ, A.C.; JONES, G.S.; JONES, H.W. Fertility following bilateral ova- rian wedge resection: a critical analysis of 90 consecutive cases of the polycystic ovary syndrome. Fertil Steril 1981; 36: $320-325$.

6. KISTNER R.W. Peri-tubal and peri-ovarian adhesions subst quent to wedge resection of the ovaries. Fertil Steril 1969; 20: 35-42.

7. COHEN M.R. Laparoscopy, culdoscopy and gynecography; Technique and atlas. Philadelphia, 1970 W.B. Saunders Company, p. 97. 
8. BUTTRAM V.C.; VAQUERO C. Post-ovarian wedge resection adhesive disease. Fertil Steril 1975; 26: 874 - 876.

9. WEINSTEIN:D.; POLISHUK W.Z. The role of wedge resection of the ovary as a cause for mechanical sterility. Surgery Ginecol Obstet 1975; 141: $417-418$.

10. TOAFF R.; TOAFF M.E.; PEYSER M.R. Infertility following wedge resection of the ovaries. Am J. Obstet Gynecol 1976; 124: 92 - 96.

11. LOY R.; SEIBEL M.M. Evaluation and therapy of polycistic ovarian syndrome. Endocrinol Metab Clin North America 1988; vol 17 (4): $785-813$.
12. GOMEZ G.; LONDOÑO R.D.; COHEN P. Inducción de la ovulación: Clomifeno, Rev Col Obst Ginec, 1986, 6: 409 - 418.

13. PEREZ E.; PARADA C.; DUARTE A.; LUZARDO L.F. Tumores de ovario. Rev Col Obst Ginec, 1975, 6: 369 - 380.

14. MORENO A. Tumores de ovario en el Hospital General de Neiva. Rev Col Obst Ginec, 1985, 2: 95 - 101.

15. RUTLEDGE F. Chap 34. Te Linde Operative Gynecology. 1985 Edit Lippincott Company, 6 edition.

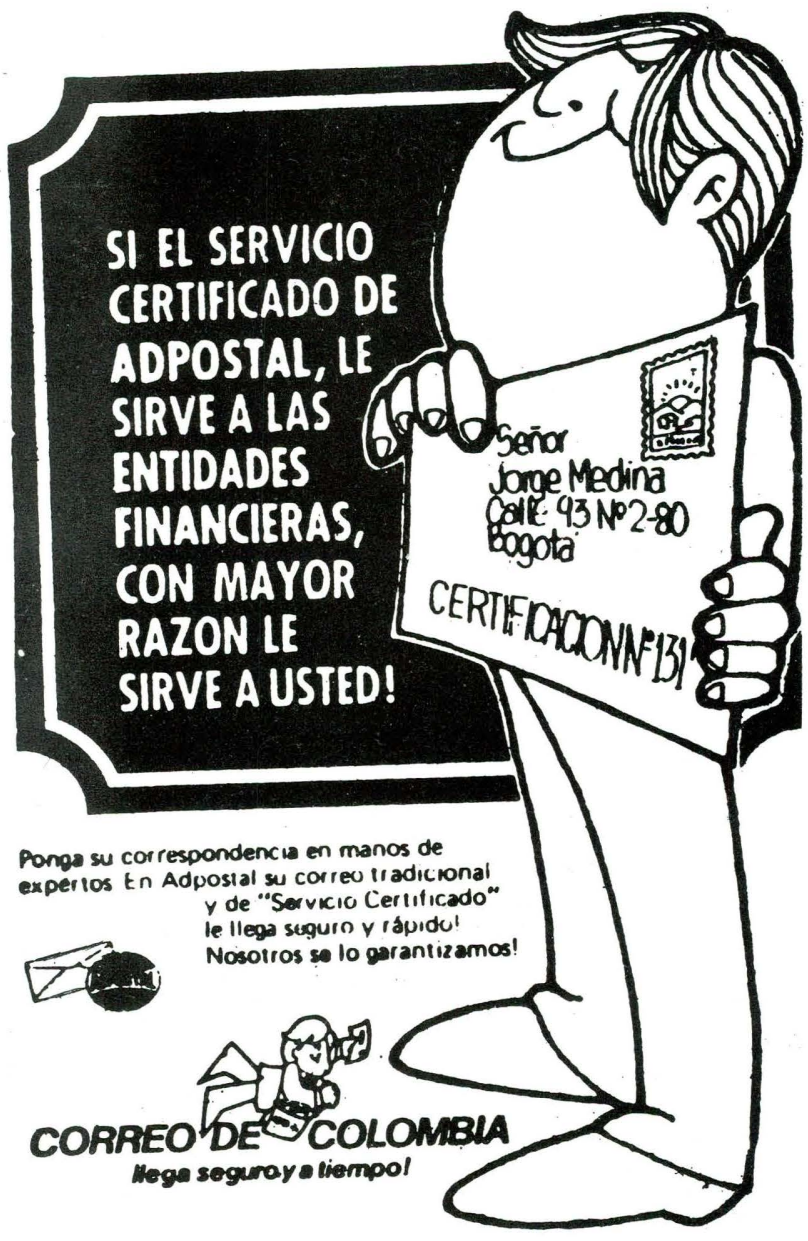

